\title{
Research on Evolutionary Mechanism of Agile Supply Chain Network via Complex Network Theory
}

\author{
Nai-Ru Xu, ${ }^{1}$ Jia-Bao Liu, ${ }^{2}$ De-Xun Li, ${ }^{3}$ and Jun Wang ${ }^{4}$ \\ ${ }^{1}$ The School of Finance and Economics, Anhui Wonder University of Information Engineering, Hefei 231201, China \\ ${ }^{2}$ The Department of Public Courses, Anhui Xinhua University, Hefei 230088, China \\ ${ }^{3}$ School of Political Science and Public Administration, Wuhan University, Wuhan 430072, China \\ ${ }^{4}$ School of International Business Administration, Shanghai University of Finance and Economics, Shanghai 200433, China \\ Correspondence should be addressed to Jia-Bao Liu; liujiabaoad@163.com
}

Received 27 October 2015; Revised 23 December 2015; Accepted 27 December 2015

Academic Editor: Young Hae Lee

Copyright (c) $2016 \mathrm{Nai}-\mathrm{Ru} \mathrm{Xu}$ et al. This is an open access article distributed under the Creative Commons Attribution License, which permits unrestricted use, distribution, and reproduction in any medium, provided the original work is properly cited.

\begin{abstract}
The paper establishes the evolutionary mechanism model of agile supply chain network by means of complex network theory which can be used to describe the growth process of the agile supply chain network and analyze the complexity of the agile supply chain network. After introducing the process and the suitability of taking complex network theory into supply chain network research, the paper applies complex network theory into the agile supply chain network research, analyzes the complexity of agile supply chain network, presents the evolutionary mechanism of agile supply chain network based on complex network theory, and uses Matlab to simulate degree distribution, average path length, clustering coefficient, and node betweenness. Simulation results show that the evolution result displays the scale-free property. It lays the foundations of further research on agile supply chain network based on complex network theory.
\end{abstract}

\section{Introduction}

Choi et al. view the supply chain network as complex adaptive system (CAS) firstly, introducing a method of managing supply chain network in the view of the complex adaptive system [1]. Surana et al. systematically and formally apply the CAS to solve the problems in the context of the supply chain network and present that the appearance of information technology makes supply chain acquire complex self-organization ability like biological system in the trend of atomization of supply chain market and atomization of organization entity [2]. Wycisk et al. summarize the attribute similarity and the behavior similarity between the supply network and the CAS. The attribute similarity includes heterogeneity, interaction, independency, and learning ability of agency. The behavior similarity includes self-organizations, dissolution zone (the zone between ordered border and disordered border), and coevolution. He also deems that logistics system is not self-organization system absolutely. The parts of selforganization in logistics system mainly come from the parts of decentralized decision. Furthermore, the development of information and telecommunication technology made equipment and products intelligent, such as intelligent tag and RFID. The intelligent equipment and products are called intelligent units. The intelligent units improve the selforganization ability of the supply chain network including logistics system [3]. Hearnshaw and Wilson use the complex network model to mirror the attributes of the supply chain network in reality and deem that the supply chain network in reality is similar to the scale-free network and has its characteristics, such as short characteristic path length, high clustering coefficient, and power law link distribution [4]. At this time, the complex network characteristics of the supply chain network have been explained systematically and clearly. The contribution made by those authors and experts leads to the fact that people make agreement on applying the complex network theory into the supply chain research [5-8].

The widespread application of the Internet technology as the representative of the rapid development of information technology and the development of economic globalization 
make the traditional business model face new challenges and make business outsourcing caused by the horizontal and vertical cooperation become more popular. These make supply chain management become more important. At the same time, fluctuations in market demand, such as the emergence of new products and services, shortening product life cycle, and product demand growth or atrophy, and changes in the external environment, such as natural disasters, strikes, war, and terrorism, all require that the supply chain becomes more agile than ever in order to detect opportunities and threats in the market and changes in the internal and external environment in time and then quickly make decisions and take actions to deal with them. This kind of quick response capability is the main feature of the agile supply chain. The agile supply chain emphasizes the quick response capability of the supply chain which is different from resilient supply chain. The latter emphasizes the capability that the resilient supply chain can quickly return to the original state after being disturbed, possessing passive preventive property, while the agile supply chain actively detects changes and fluctuations and immediately takes measures to deal with them in time, possessing active property.

In the agile supply chain research, the agile supply chain modeling is an important approach. Lau et al. use virtual agent technology to design and develop the infrastructure frame of the agile supply chain system which improves the forecasting ability of supply chain [9]. Lou et al. deem that the supply chain is composed of a series of agents. The function of supply chain management system is to coordinate these agents. They present the agile supply chain management framework based on multiagent theory to define the function and responsibility of the agent in order to coordinate supply chain operation by improving the efficiency and effect of the internal division of labor in the agile supply chain [10]. Kim combines object-oriented method with analytic hierarchy process to design the agile supply chain business model and presents the steps of identifying and expressing the business rules in the object-oriented model. This method emphasizes the role of business rules in maintaining the operation of agile supply chain [11]. Z. X. Li and R. Li make the static structure analysis and the dynamic behavior analysis on supply chain system based on the Petri net theory in order to achieve the integral integration and coordinated control of each node in the supply chain finally [12]. However, this method does not refer to the supply chain network evolution analysis. The agile supply chain management system model based on coordination decision center and workflow management technology proposed by Cheng [13] and the agile supply chain management system operation pattern guided by integrated information flow proposed by Gu and Qiao [14] both belong to a kind of static study in the view of the basic structure framework.

In summary, the current modeling research does not involve the dynamic evolution mechanism of the agile supply chain network. However, there have been some research results on the evolution mechanism of the supply chain network based on complex network theory.

In the present results of evolution mechanism research of the supply chain network based on complex network theory, there are researches on different types of supply chain network, such as supply chain directed network [15], layered supply chain network [16], cluster supply chain network [17], and disruption supply chain [18]. Besides, there are researches on uncertainty [19], robustness [20]. There are researches on evolution of supply chain network in different evolution conditions, such as the supply chain with leaving nodes and entering nodes existing together [21], the supply chain network based trading volume [22]. However, the current research has not referred to the evolution mechanism of the agile supply chain based on complex network theory which can describe the motivation and features of dynamic evolution process of the agile supply chain. This paper makes research on the evolution mechanism of the agile supply chain based on complex network theory.

The remainder of the paper is organized as follows. We first introduce related concepts on the complex network and the agile supply chain network and then present the evolutionary mechanism of agile supply chain network based on complex network theory and make simulation analysis and then discuss the features of scale-free networks generated with the evolutionary mechanism model. Finally, the paper will give a conclusion and discuss future research directions. The schematic diagram of technology roadmap is as shown in Figure 1.

\section{Complex Network and Agile Supply Chain Network}

2.1. The Concept of Complex Network and Statistical Characteristics. The complex network is composed of a cluster of nodes which are independent and connect with each other and is a type of network with high complexity. A node represents an individual; the edge represents the link between nodes. X. S. Qian gives relatively strict definition for complex network: a type of network obtaining the following parts or all of characteristics: self-organization, being self-similar, attractiveness, small world, and being scale-free. The scalefree network is a kind of typical complex network. This model is proposed by Barabási and Albert [22] according to the phenomenon that degree distribution of networks in reality has the attribute of power law distribution. The model is called BA model [23].

In the BA model, the evolution process of network has two driver factors: growth and preference link. Based on these two key driver mechanisms, the algorithm of the model is shown as follows.

Growth. The initial state of network has few nodes, $m_{0}$; on each time step, a new node is introduced to the network; at the same time, this new node connects with existing nodes.

Preference Link. There is preference when a new node chooses other nodes to connect. The ratio of choosing node $i$ is the proportion to the degree of this node. Consider

$$
\Pi_{i}=\frac{k_{i}}{\sum_{j} k_{j}} .
$$




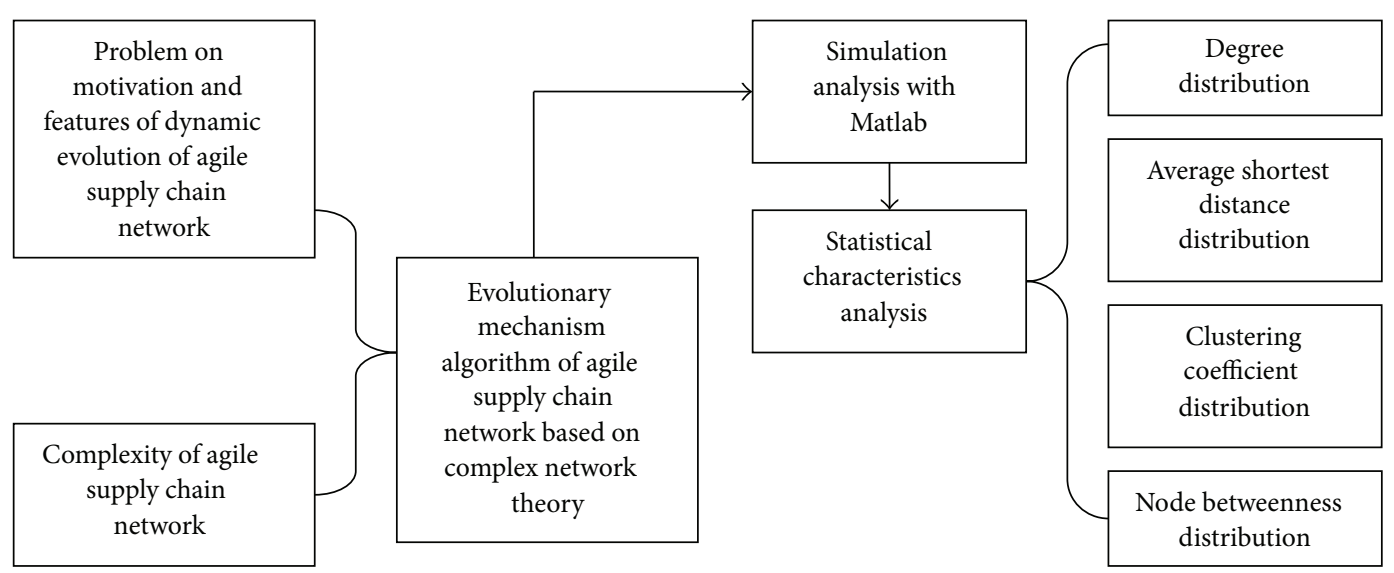

FIGURE 1: The schematic diagram of technology roadmap in the research.

After $t$ time step, the scale of the network changes, and the number of nodes becomes $N=m_{0}+t$; the number of edges is $m t$. The degree distribution of the scale-free network in BA model follows the power law distribution: $P(k)=2 m^{2} k^{-3}$.

In order to reflect the topological structure of complex network, the basic indicators are usually used; there are degree distribution, average path length, clustering coefficient, and node betweenness [24].

2.1.1. Degree Distribution. Degree $k_{i}$ delegates the number of edges which connect node $i$. the bigger the degree is, the more important the node is. The formula of $k_{i}$ is shown as follows:

$$
k_{i}=\sum_{j \in N} a_{i j}
$$

Degree distribution of nodes in the network can be described by $p_{k} ; p_{k}$ is the probability that the degree value of a node being chosen randomly is $k$. In a statistical sense, $p_{k}$ is the ratio of the number of nodes whose degree value is $k$ to the total number of nodes in the network:

$$
P(k)=\frac{n_{k}}{N} .
$$

$n_{k}$ indicates the number of nodes whose degree value is $k$; $N$ is the total number of nodes in the network.

2.1.2. Average Path Length. Average path length is the average shortest distance between all pairs of nodes in the network. The shortest path is defined as the distance $d_{i j}$ between node $i$ and node $j$. The formula is shown as

$$
L=\frac{1}{(1 / 2) N(N-1)} \sum_{i \geq j} d_{i j} .
$$

$N$ indicates the total number of nodes in the network. Average path length includes the distance from the node to itself (the value is 0 ).
2.1.3. Clustering Coefficient. Assume that there are $k_{i}$ nodes connecting with node $i$ in the network; these $k_{i}$ nodes are called neighbors of node $i$. The biggest number of edges is likely $k_{i}\left(k_{i}-1\right) / 2$ among the $k_{i}$ nodes. The clustering coefficient $C_{i}$ is defined as the ratio of the real number of the edges $E_{i}$ among these $k_{i}$ nodes to the largest number of edges, $k_{i}\left(k_{i}-1\right) / 2$. Consider

$$
C_{i}=\frac{2 E_{i}}{k_{i}\left(k_{i}-1\right)} .
$$

2.1.4. Betweenness. Betweenness includes node betweenness and edge betweenness. It is a global variable and reflects the influence and importance of node or edge in the whole network. If there are $M$ shortest paths among nodes and $m$ shortest paths of them going through node $i$, so the contribution value of betweenness is $m / M$ which is made by node $i$ to node pairs. Sum up all contribution value of node $i$ to all other nodes and then use the number of total node pairs to divide the sum of all contribution value. At last, the obtained quotient is the betweenness of node $i$. According to the same means of definition, edge betweenness is the ratio of the number of the shortest paths going through the edge to the shortest paths of all node pairs. A lot of research shows that there is a strong correlation between the node betweenness and the node degree.

The betweenness formula of node $v$ is

$$
g(v)=\sum_{s \neq t, s \neq v} \frac{\sigma_{s t}(v)}{\sigma_{s t}} .
$$

$\sigma_{s t}$ is the number of the shortest paths from node $s$ to node $t$ (node $s$ and node $t$ refer to two different nodes randomly); $\sigma_{s t}(v)$ is the number of the shortest paths from node $s$ to node $t$ which go through node $s$ to node $v$.

2.2. Concept and Characteristics of Agile Supply Chain. Agile supply chain is focused on improving responsiveness and flexibility and is able to respond and react quickly and effectively to changing markets [25]. The characteristics of agile supply chain have four items [24]. 
2.2.1. Market Sensitivity. Market sensitivity refers to the ability of mastering and making respond to real demand. The production plan is driven by market demand, not by the demand prediction which is made based on statistical analysis on historical sale data. The enterprise can respond to the customized demand through supply chain responsiveness at the fastest speed, and the whole supply chain remains dynamic refreshment in order to enable the core enterprise to satisfy customer demand.

2.2.2. Virtuality. Due to the technical and artificial factors, the demand information could be distorted easily when it flows from the downstream to the upstream. This distortion leads to deviation between production plan of production enterprises and supply preparation plan of suppliers and thus forms the bullwhip-effect; that is to say, a small change of actual demand of end users can cause a large scope change of purchasing quantity of upstream enterprises. The agile supply chain uses information technology to share the data among the phrases of up- and downstream in the supply chain which can construct a virtual supply chain so as to diminish the bullwhip-effect problem.

2.2.3. Process Integration. Process integration means cooperation between partners, including cooperative work, common product development, general purpose system, and information sharing among nodes in supply chain. Through this process integration, the enterprise can be more concerned about the development of core competencies on one hand and integrate the suppliers in upstream and customers in downstream into the strategic planning of the enterprise on the other hand in order to achieve the optimal allocation of internal and external resources for enterprises.

2.2.4. Based on Network. The supply chain network is a union of the partners who combine together like a network. Enterprises no longer participate in competition in the form of individual one but in the form of supply chain network. Eventual winners will be the enterprises which better organize and coordinate the cooperative partnership so as to provide the better, the faster, and the closer service to the end users through the supply chain network.

\subsection{Complexity Analysis of the Agile Supply Chain Network}

2.3.1. Integration Complexity. Synergy function in the organizational and the managerial activities is the most frequent one in the agile supply chain network. Collaborative requirements are the highest ones; the emergence phenomenon is the most significant. Therefore, integration complexity is mainly divided into organizational complexity and managerial complexity. The organizational complexity is mainly influenced by the certainty and quantity of supply chain structure, scale, and internal relationship. Managerial complexity is a complex phenomenon in the supply chain design, adjustment, reengineering, and management process.
2.3.2. Operational Complexity. Operational process of the agile supply chain network is the process of dynamic management of the connection, the distribution, the layout, the goal, and so forth among the various elements, is the process of planning and coordinating information, nodes, paths, and technologies needed by supply chain, and is the collaborative process of planning and utilizing all kinds of soft resource. It reflects dynamic complexity of the agile supply chain network.

2.3.3. Manufacturing Complexity. Manufacturing complexity in the agile supply chain network refers to some nonlinear and unpredictable phenomena emerging in the process of product design and production.

2.3.4. Environmental Complexity. Environmental changes will lead to the corresponding changes in the agile supply chain network. The speed and the extent of environmental changes may cause the unpredictable changes of the internal factors of the agile supply chain network and cause quantity and quality relationship among elements to produce the corresponding nonlinear changes, eventually leading to the increased complexity of the agile supply chain network.

\section{Evolutionary Mechanism of Agile Supply Chain Network Based on Complex Network Theory and Simulation Analysis}

3.1. Evolutionary Mechanism. The agile supply chain network can be described as a set $G=(N, E)$, which contains member enterprises $(N)$ and a group of edges with weight $(E)$. The weighted adjacency matrix $W$ is used to delegate the agile supply chain network. Matrix element $w_{i j}\left(w_{i j} \geq 0\right)$ represents average business response speed between enterprise $i$ and enterprise $j . w_{i j}=0$ means that there is no business link between enterprise $i$ and enterprise $j$. Because of bidirectional nature of logistics flow, capital flow, and information flow in business activities, it is assumed that agile supply chain network is undirected network.

Definition 1. The node strength of node enterprise $i, s_{i}$, is

$$
s_{i}=\sum_{j \in N_{i}} w_{i j} .
$$

$N_{i}$ refers to the nearest neighbor set of node enterprise $i$. The node strength not only considers the number of the nearest neighbors of node enterprise but also considers the weights of business links between this enterprise and its nearest neighbors and is the integrated embodiment of local information of this node enterprise.

Definition 2. The distance between node enterprise $i$ and node enterprise $j$ can be expressed as

$$
L_{i k}=\frac{w_{i j}+w_{j k}}{w_{i j} w_{j k}}
$$


TABLE 1: Statistical criteria of choosing partners of Chinese enterprises.

\begin{tabular}{lcccccc}
\hline Selection criteria & Quality & Price & $\begin{array}{c}\text { Delivery } \\
\text { lead time }\end{array}$ & $\begin{array}{c}\text { Variety } \\
\text { flexibility }\end{array}$ & $\begin{array}{c}\text { Lead time and } \\
\text { price compromise }\end{array}$ & $\begin{array}{c}\text { Lead time and batch } \\
\text { compromise }\end{array}$ \\
\hline Percentage (\%) & 98.5 & 92.4 & 69.7 & 45.5 & 30.3 & 21 \\
\hline
\end{tabular}

Let $l_{i k}=1 / L_{i k}=w_{i j} w_{j k} /\left(w_{i j}+w_{j k}\right)$. In the formula, the node enterprise $i$ makes connection with node $j$ and node $k$ by means of two edges with weight of $w_{i j}$ and $w_{j k}$, respectively. We can obtain the distance between any two node enterprises based on the formula and further get the shortest distance between any member enterprises in the agile supply chain network and the average shortest distance of the agile supply chain network.

Definition 3. The definition of clustering coefficient of node enterprise $i$ is

$$
C_{H, i}^{w}=\frac{\sum_{j, k} w_{i j} w_{j k} w_{k i}}{\max _{j}\left(w_{i j}\right) \sum_{j, k} w_{i j} w_{k i}} .
$$

Combine these three basic concepts coming from the weighted network evolution model with the unique attributes of the agile supply chain network evolution; we can get the rule of evolution of edge weight in the agile supply chain network model.

Edge weight evolves in accordance with the processed business volume per unit time between node enterprises (response speed); the newly added business volume per unit time transforms to edge weight conversely and drives the network evolution again.

The basic evolution mechanism of the agile supply chain network model is shown as the follows:

(1) Evolution stems from the complete network with $m_{0}$ node enterprises. The weight of each edge is initialized to $w_{i j}=f(0)$.

(2) At the $t$ time step, add a new node enterprise $n$ into the network, this new node makes connection with $m\left(m \leq m_{0}\right)$ old nodes, and the weight of link is $g(t)$. The probability of the new node linking with node $i$ is shown as

$$
\Pi_{n \rightarrow i}=p \frac{s_{i}}{\sum_{j} s_{j}}+(1-p) \frac{l_{n i}}{\sum_{j} l_{n j}} .
$$

$s_{i}$ is the node strength of node $i$ and $l_{n i}$ the reciprocal of the shortest distance between node $n$ and node $i$; that is $l_{n i}=1 / L_{n i} \cdot p$ is the factor of proportionality. This formula illustrates that the new node makes connection with the existing nodes under the premise of taking into account the response speed which reflects the business processing ability per unit time, the node with the faster response speed is preferred, and at the same time, taking into account the average shortest path distance between the node preparing to be linked and the new one, the node with closer distance is preferred.
(3) The formula of edge weight evolution is

$$
f(t)=f(t-1)+g(t) .
$$

(4) The factor of proportionality is $p$.

According to the survey data given by the supply chain management research group in School of Management, Huazhong University of Science \& Technology, when Chinese enterprises choose partners, the factors considered by them are shown as in Table 1.

Separate delivery lead time, price compromise, and batch compromise being related with lead time from the factors and calculate the ratio of the sum of three factors to the sum of all factors. The ratio can be the factor of proportionality $(1-p)$ which reflects time influence of the average shortest path and then get $p=0.72$.

3.2. Simulation Analysis. We use Matlab R2014a software. The computer is configured as AMD processor. The dominant frequency is $2.50 \mathrm{GHz}$. Set $m_{0}=10, m=3, p=0.72$, and the network size $N=1000$; the processed business volume per unit time (edge weight) can be set randomly. The evolution stems from the agile supply chain network with 10 node enterprises, each one of which makes business with all other node enterprises. In other words, this network is a complete network. The newly added node connects with three old nodes in the network. Evolution scale grows up to the 1000 nodes in the network. Observe the relationship between the network size with degree distribution, average shortest distance, clustering coefficient, and node betweenness.

3.2.1. Degree Distribution. Simulation results in Figure 2 show that the curve of degree distribution possesses the feature of power law distribution, and most nodes have small degree value; only few nodes have big degree value. There are seldom nodes whose degree value falls in the interval $(10,18)$; their emergency probability is very low. There are many nodes whose degree value falls in the interval $(0,10)$, and emergency probability is high. These features illustrate that the model can obviously reflect the scale-free feature of the agile supply chain network.

3.2.2. Average Shortest Distance Distribution. Figure 3 shows that the average path length between node enterprises in the agile supply chain network grows with the growth of network size, but the total length is not large. It is related with virtuality of dynamic association in the agile supply chain network. In the sense of dynamic association, the nodes constantly reconfigure cooperative relationships between themselves according to the business demand in the internal 

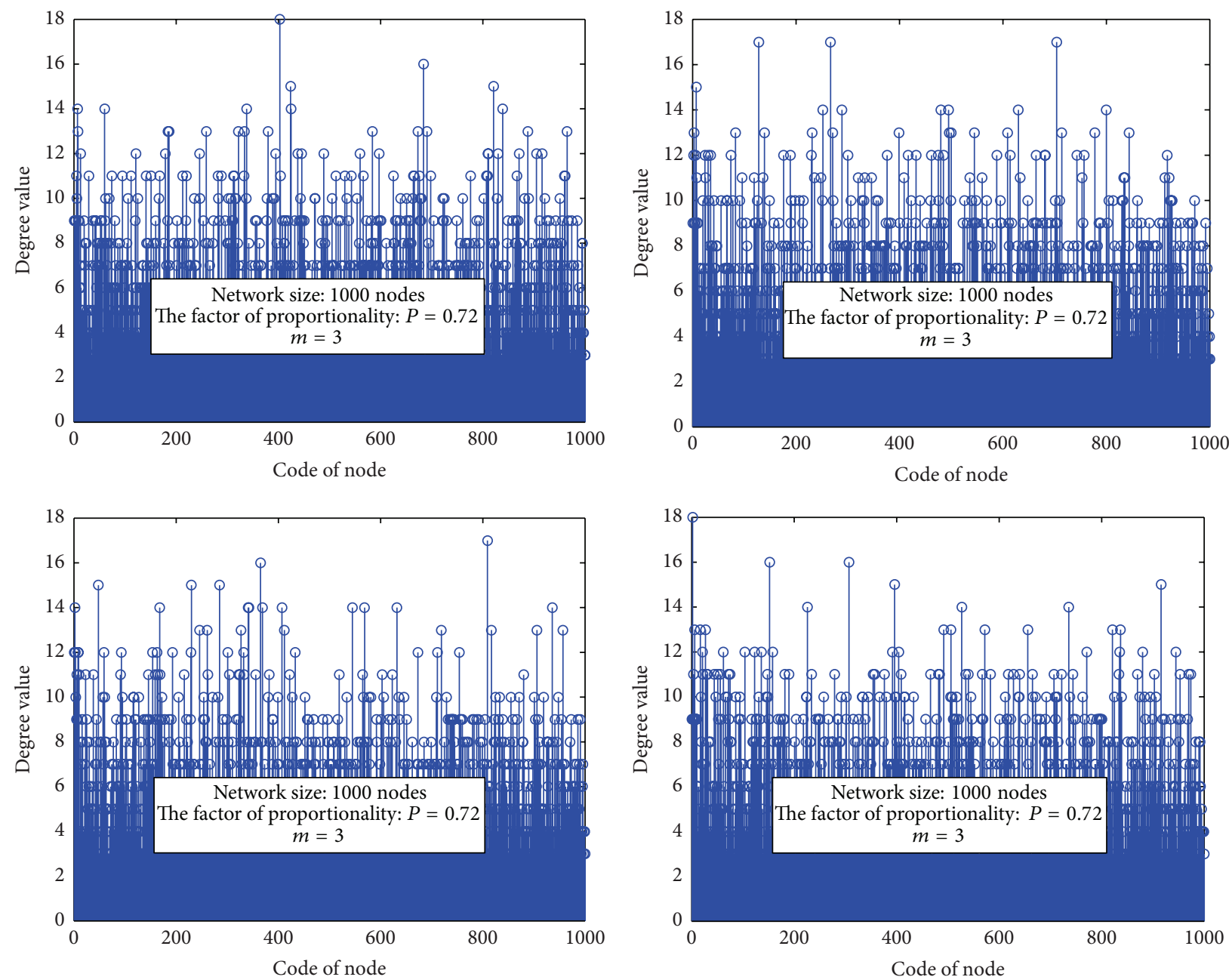

(a)

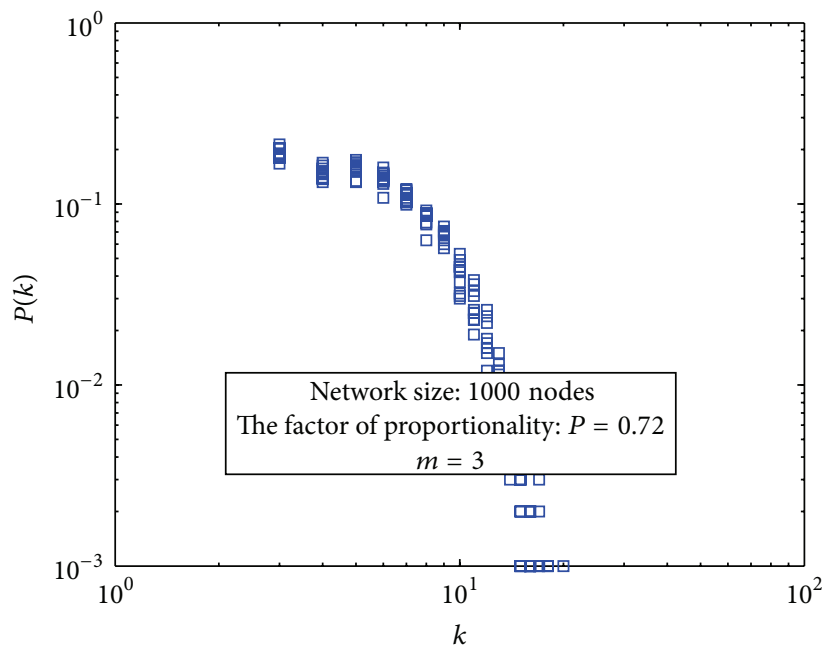

(b)

FIgURE 2: Degree distribution. 


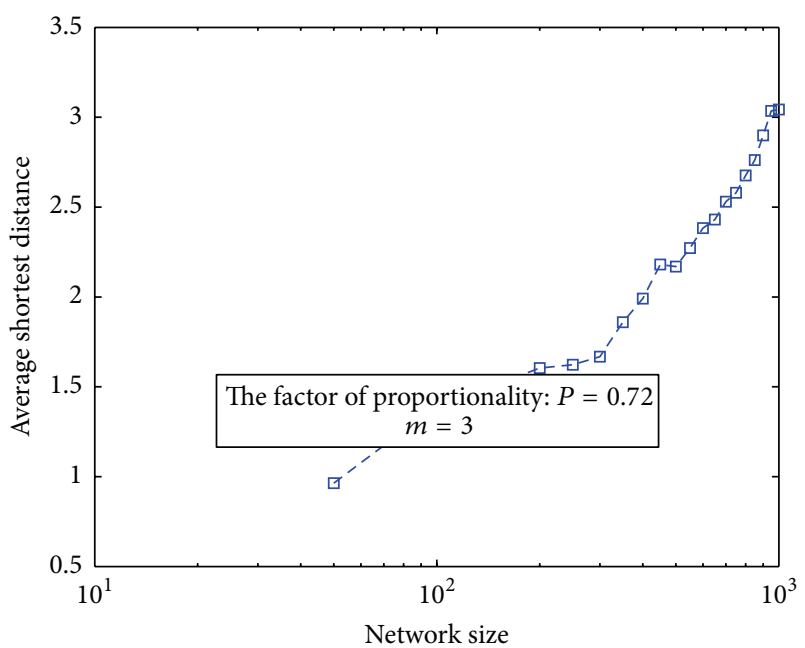

FIGURE 3: Average shortest distance distribution.

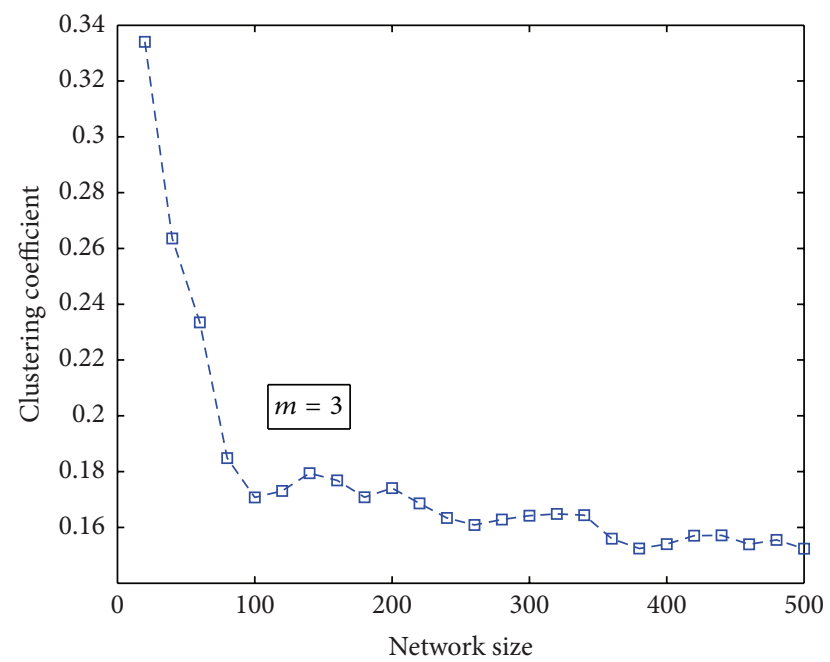

FIgURE 4: Clustering coefficient distribution.

and external environment in order to form the new supply chain network. The nodes keep close relationship with each other by means of information technology and information system, which leads to relatively short characteristic path length.

3.2.3. Clustering Coefficient Distribution. Figure 4 shows the clustering coefficient of the agile supply chain network falls in the interval $(0.1,0.4)$. Clustering level is not high and gradually decreases with the growth of network size, presenting long tail distribution. The reason is that the agile supply chain network takes on network distribution. The enterprises prefer to finish the business process by means of horizontal cooperation rather than complete all tasks by means of vertical integration. In this way, the node enterprises can utilize core competitive power fully, as well as by means of external resources, and enforce agility and flexibility of supply chain. Thus, the agile supply chain network can adjust the composition of node enterprises, business progress, and organization structure, in order to actualize dynamic reconfigure of the agile supply chain network.

3.2.4. Node Betweenness Distribution. Figure 5 shows that the node betweenness value falls in the interval $(0,0.12)$, most nodes have small betweenness value, only few nodes have large betweenness value, and the curve of betweenness distribution presents power law distribution. It is consistent with the low clustering coefficient in the agile supply chain network.

The simulation analysis of these four indicators illustrates that the main statistical features of evolution mechanism of the agile supply chain network can well reflect the features of scale-free network.

\section{Conclusions}

From the perspective of network structure, the paper systematically and dynamically analyzes the evolutionary process of the agile supply chain network by means of the evolution mechanism model, reveals agility motivation for individual decision making and network characteristics of evolution results in the agile supply chain network evolution, and also analyzes the mechanism of making decision and action of individuals in self-organization movement improving the agility of the whole supply chain network. This provides a reference for further optimization of the agile supply chain network.

The paper explains the adaptability of complex network theory to the research on the agile supply chain network and analyzes the complexity of the agile supply chain network based on the introduction of basic concepts of complex network and agile supply chain network. Then, the paper designs the evolution mechanism model of the agile supply chain network based on complex network theory. In the evolution mechanism, node enterprise makes a decision on connection according to two factors. One is the responsive ability; the other is distance. They construct the link criterion of agility in the agile supply chain network. After that, the simulation analysis is presented. Simulation results show that the agile supply chain network obviously possesses the features of scale-free network.

In the future research, we can use complex network theory to study the robustness of the agile supply chain network generated by the evolution mechanism designed by the paper, which is the ability of continually maintaining the operation when the supply chain network is disturbed. Besides, we can also make research on risk management consisting of risk identification, risk diffusion, and control in the agile supply chain network based on the evolution mechanism and study the network efficiency of the agile supply chain network which is generated by the evolution mechanism and other related fields.

\section{Conflict of Interests}

The authors declare that there is no conflict of interests regarding the publication of this paper. 

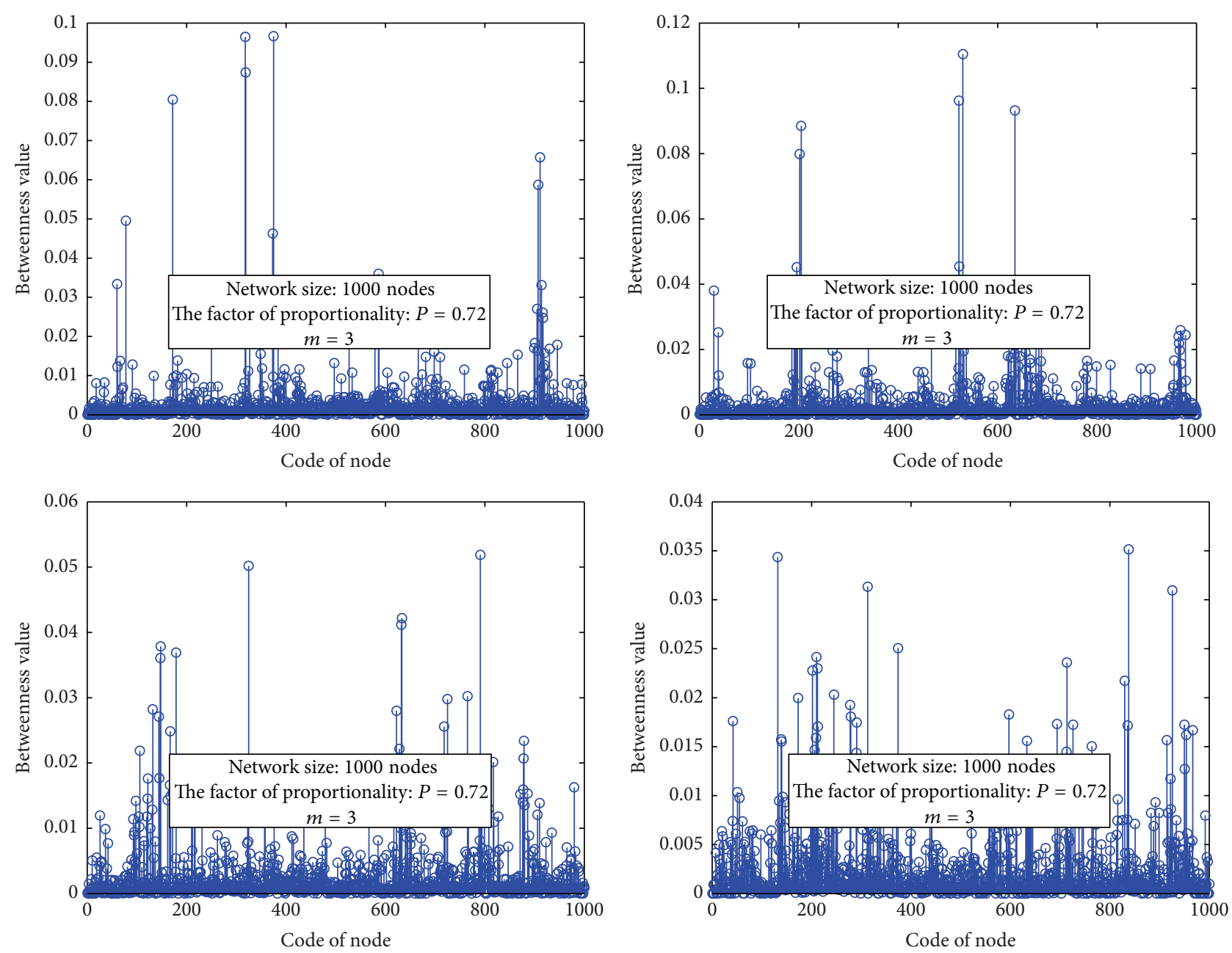

(a)

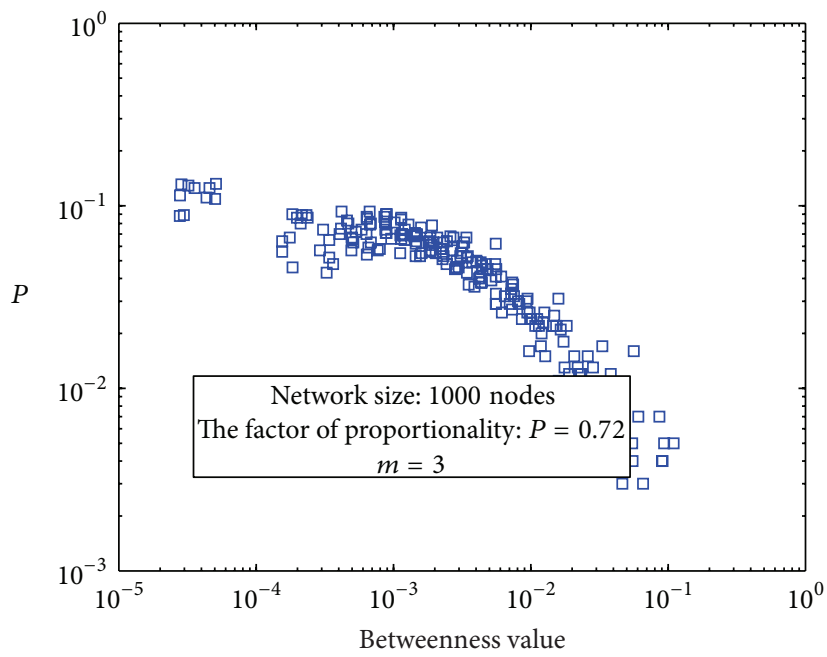

(b)

FIGURE 5: Node betweenness distribution. 


\section{Acknowledgments}

The work of Nai-Ru Xu was supported in part by the Key Project of the Special Foundation for Young Scientists of Anhui Province of China under Grant no. 2013SQRW090ZD. The work of Jia-Bao Liu was supported in part by the Natural Science Foundation of Anhui Province of China under Grant no. KJ2013B105, the Natural Science Foundation for the Higher Education Institutions of Anhui Province of China under Grant no. KJ2015A331.

\section{References}

[1] T. Y. Choi, K. J. Dooley, and M. Rungtusanatham, "Supply networks and complex adaptive systems: control versus emergence," Journal of Operations Management, vol. 19, no. 3, pp. 351366, 2001.

[2] A. Surana, S. Kumara, M. Greaves, and U. N. Raghavan, "Supply chain networks: a complex adaptive systems perspective," International Journal of Production Research, vol. 43, no. 20, pp. 4235-4265, 2005.

[3] C. Wycisk, B. McKelvey, and M. Hülsmann, "'Smart parts' supply networks as complex adaptive systems: analysis and implications," International Journal of Physical Distribution \& Logistics Management, vol. 38, no. 2, pp. 108-125, 2008.

[4] E. J. S. Hearnshaw and M. M. J. Wilson, "A complex network approach to supply chain network theory," International Journal of Operations \& Production Management, vol. 33, no. 4, pp. 442469, 2013.

[5] K. Zhao, A. Kumar, T. P. Harrison, and J. Yen, "Analyzing the resilience of complex supply network topologies against random and targeted disruptions," IEEE Systems Journal, vol. 5, no. 1, pp. 28-39, 2011.

[6] S. I. Mari, Y. H. Lee, M. S. Memon, Y. S. Park, and M. Kim, "Adaptivity of complex network topologies for designing resilient supply chain networks," International Journal of Industrial Engineering, vol. 22, no. 1, pp. 102-116, 2015.

[7] J. L. Guo, "Bilateral power-law distribution model of supply chain network," Acta Physica Sinica, vol. 55, no. 8, pp. 3916-3921, 2006.

[8] N. R. Xu and J. B. Liu, "Research on evaluation on agility of agile supply chain network based on complex network theory," Mathematical Problems in Engineering, vol. 2015, Article ID 707459, 10 pages, 2015.

[9] H. C. W. Lau, C. W. Y. Wong, K. F. Pun, and K. S. Chin, "Virtual agent modeling of an agile supply chain infrastructure," Management Decision, vol. 41, no. 7, pp. 625-634, 2003.

[10] P. Lou, Z.-D. Zhou, Y.-P. Chen, and W. Ai, "Study on multiagent-based agile supply chain management," International Journal of Advanced Manufacturing Technology, vol. 23, no. 34, pp. 197-203, 2004.

[11] J. Kim, A Methodology to Design an Agile Supply Chain Business Model Using Object-Oriented Approach and Analytic Hierarchy Process, The University of Texas At Arlington, 2004.

[12] Z. X. Li and R. Li, "Modeling and analysis of agile supply chain based on hierarchical petri net," Journal of Tianjin University of Science \& Technology, vol. 25, no. 6, pp. 58-61, 2010.

[13] T. M. Cheng, "Study of the model of agile supply chain management," in Advances in Multimedia, Software Engineering and Computing. Volume 2, D. Jin and S. Lin, Eds., vol. 129 of
Advances in Intelligent and Soft Computing, pp. 583-588, Springer, 2011.

[14] Z. Q. Gu and Z. Q. Qiao, "Agile supply chain management pattern guided by the information flow," Information Science, vol. 27, no. 8, pp. 1182-1185, 2009.

[15] H. Liu, G. G. Zhou, and P. H. Fu, "Local evolving model research of layered supply chain complex networks," Computer Science, vol. 40, no. 2, pp. 270-227, 2013.

[16] P. H. Fu, L. Jin, and Y. C. Liu, "Cluster supply chain network evolving model based on degree and path preferential attachment," Operations Research and Management Science, vol. 22, no. 1, pp. 120-125, 2013.

[17] B. X. Zhu and Y. H. Hu, "Study on supply chain disruption management based on complex network theory," Logistics Technology, vol. 26, no. 1, pp. 147-150, 2007.

[18] X. Fan, J. H. Ma, and Y. Xiu, "Analysis of uncertainty in complex supply chain networks," Complex Systems and Complexity Science, vol. 3, no. 3, pp. 20-25, 2006.

[19] X. Xu, R. W. Li, X. L. Wu, and H. X. Liu, "Research on robustness of complex supply chain networks based on network quality control," China Mechanical Engineering, vol. 23, no. 8, pp. 941946, 2012.

[20] G. Li and D. Z. Zhao, "A Study on the Scale-free characteristics of supply chain network," Industrial Engineering Journal, vol. 15, no. 1, pp. 28-32, 2012.

[21] N. R. Xu and J. B. Liu, "A literature review on supply chain network research based on complex network theory," Journal of Chongqing Technology and Business University, vol. 32, no. 5, pp. 36-43, 2015.

[22] A.-L. Barabási and R. Albert, "Emergence of scaling in random networks," Science, vol. 286, no. 5439, pp. 509-512, 1999.

[23] R. Albert and A.-L. Barabási, "Statistical mechanics of complex networks," Reviews of Modern Physics, vol. 74, no. 1, pp. 47-97, 2002.

[24] N. R. Xu, J. B. Liu, and B. H. Ruan, "A literature review of agile supply chain management research," Journal of Chongqing Technology and Business University, vol. 31, no. 6, pp. 62-70, 2014.

[25] C.-T. Lin, H. Chiu, and Y.-H. Tseng, "Agility evaluation using fuzzy logic," International Journal of Production Economics, vol. 101, no. 2, pp. 353-368, 2006. 


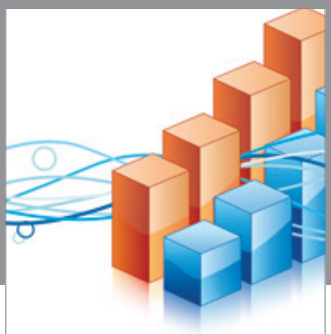

Advances in

Operations Research

vatem alat4

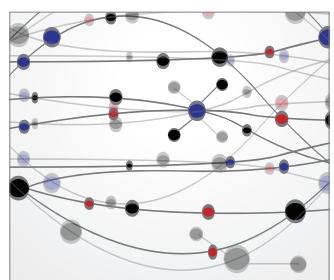

\section{The Scientific} World Journal
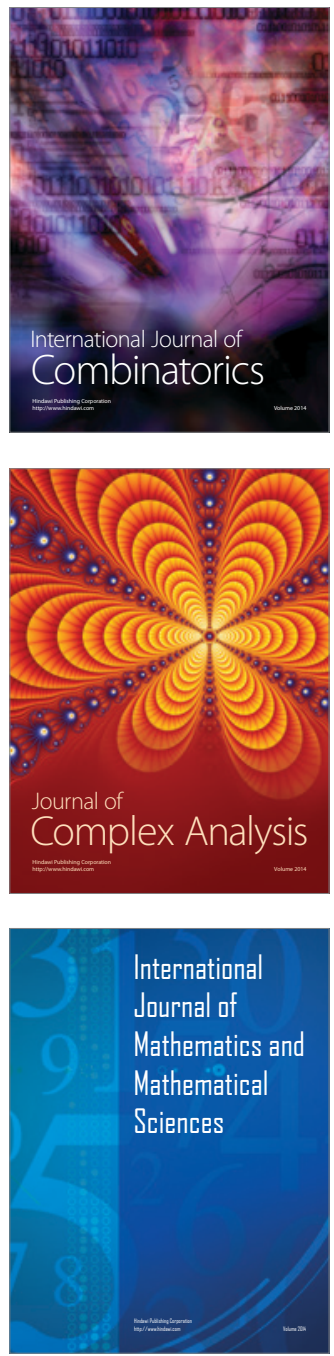
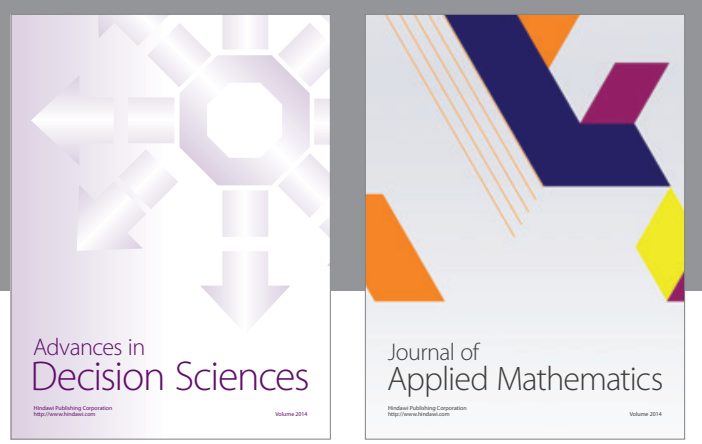

Algebra

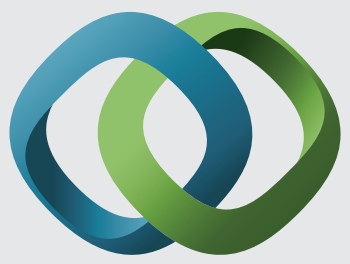

\section{Hindawi}

Submit your manuscripts at

http://www.hindawi.com
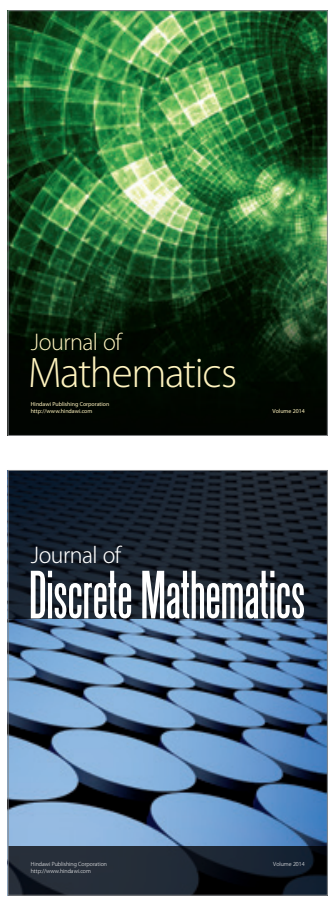

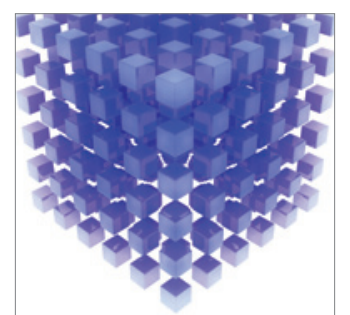

Mathematical Problems in Engineering
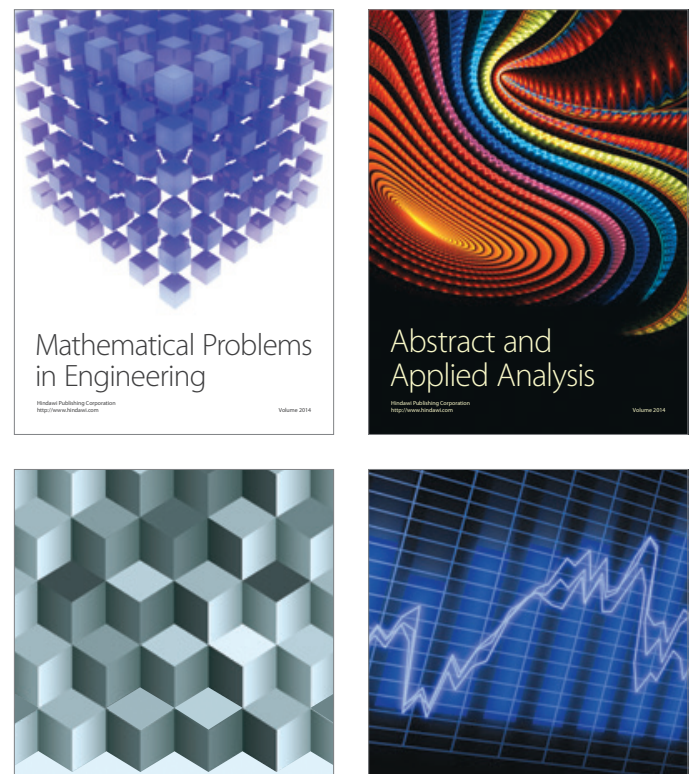

Journal of

Function Spaces

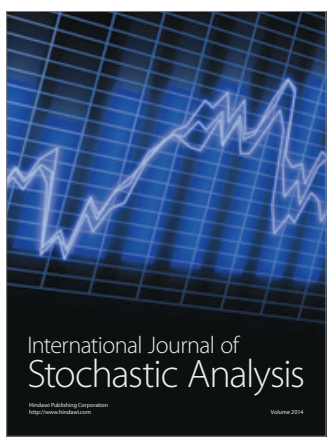

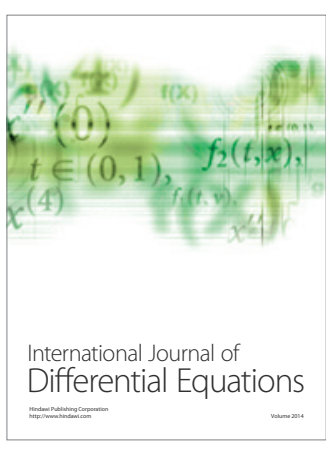
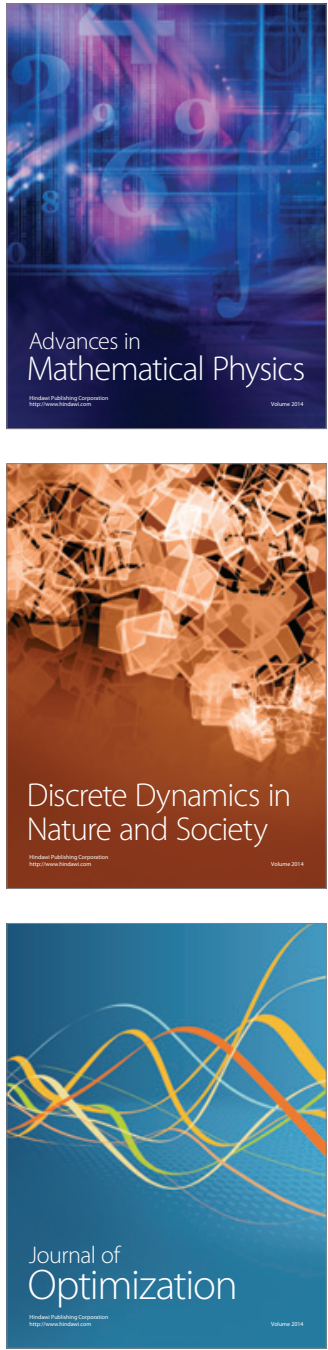